\title{
Additional Symmetries of Supersymmetric KP Hierarchies
}

\author{
Sonia Stanciu ${ }^{\star}$ \\ Physikalisches Institut der Universität Bonn, Nussallee 12, D-53115 Bonn, Germany
}

Received: 27 September 1993/in revised form: 23 March 1994

\begin{abstract}
We investigate the additional symmetries of several supersymmetric KP hierarchies: the SKP hierarchy of Manin and Radul, the $\mathrm{SKP}_{2}$ hierarchy, and the Jacobian SKP hierarchy. In all three cases we find that the algebra of symmetries is isomorphic to the algebra of superdifferential operators, or equivalently $\mathbf{S W}_{1+\infty}$. These results seem to suggest that despite their realization depending on the dynamics, the additional symmetries are kinematical in nature.
\end{abstract}

\section{Introduction}

The aim of this paper is to study the additional symmetries of the following supersymmetric extensions of the KP hierarchy: the SKP hierarchy of Manin and Radul [1], the $\mathrm{SKP}_{2}$ hierarchy [2], and the Jacobian SKP hierarchy of Mulase [3] and Rabin [4]. The additional symmetries of the KP hierarchy were first studied in [5] (although see [6]) and their algebraic structure has been recently identified with the algebra DOP of differential operators $[7,8]$. The additional symmetries of the supersymmetric KP hierarchy defined by Manin and Radul have been previously studied in [9].

Let us first briefly review what is known about the additional symmetries of the KP hierarchy. The KP hierarchy can be thought of as a dynamical system defined on a space whose functions are given by a subring of the ring $k[[x]]$ of formal power series in the variable $x$ with coefficients in the field $k$. It is defined as the universal family of isospectral deformations of a pseudodifferential operator $L=\partial+\sum_{i \geq 1} u_{\imath} \partial^{1-i}$. The evolution of $L$ is specified by a commuting family of flows $\partial_{i}$ in terms of which

$$
\partial_{i} L=-\left[L_{-}^{i}, L\right]=\left[L_{+}^{i}, L\right] .
$$

If one restricts oneself to operators satisfying $u_{1}=0$, then one can lift the KP flows to the Volterra group $G$. The Volterra group acts naturally via dressing transformations

^ e-mail: sonia@avzw02.physik.uni-bonn.de 
$L \mapsto \phi^{-1} L \phi$, where $\phi=1+\sum_{i \geq 1} v_{i} \partial^{-i} \in G$ is the dressing operator. In terms of the dressing operator, the flows of the KP hierarchy are given by

$$
\partial_{i} \phi=-\left(\phi \partial^{\imath} \phi^{-1}\right)_{-} \phi .
$$

One can write these flows in a different way by using an analogue of the Radul map [10]. Motivated originally by attempts to understand the geometric meaning of $\mathbf{W}$-symmetry, Radul introduced a homomorphism

$$
W: \mathbf{D O P} \rightarrow T_{L} \mathfrak{M},
$$

between the Lie algebra of differential operators and the Lie algebra of vector fields on the space of Lax operators by associating, to every differential operator $E$, the tangent vector

$$
W(E)=\left(L E L^{-1}\right)_{-} L
$$

at the point $L$ in the space $\mathfrak{M}$ of Lax operators. The similarity between the expression for the Radul map (1.3) and the one of the KP flows (1.2) suggests us to define a map

$$
W^{\prime}(E)=\left(\phi E \phi^{-1}\right)_{-} \phi
$$

from DOP to the Lie algebra $\mathscr{R}_{-}$of the Volterra group. The KP flows become now $\partial_{n} \phi=-W^{\prime}\left(\partial^{n}\right)=-\partial_{W^{\prime}\left(\partial^{n}\right)} \phi$, where $\partial_{W^{\prime}\left(\partial^{n}\right)}$ is then a flow on the Volterra group. The map (1.5) now translates the trivial fact $\left[\partial^{n}, \partial^{m}\right]=0$ into the commutativity of the flows $\left[\partial_{n}, \partial_{m}\right]=0$. This allows us to represent the flows in terms of an infinite set of times, $\partial_{\imath}=\frac{\partial}{\partial t_{i}}$, with $i=1,2, \ldots$. One interpretation of this feature is that every flow possesses an infinite number of symmetries given by the other flows. This interpretation begs the question whether these are all or, if on the contrary, there exist additional symmetries. Remarkably enough, it turns out that one can construct a larger family of times-dependent flows which contains as a subset the original KP flows and commute with them. This new family of flows satisfies a nonabelian algebra with respect to which the KP hierarchy forms its center. Thus we adopt here the following definition.

Definition 1.6. By (additional) symmetries of an integrable hierarchy of flows, we mean its centralizer in the algebra of times-dependent vector fields.

The fact that these symmetries contain the original hierarchy, although largely taken for granted, is only true provided the flows of the hierarchy themselves satisfy an abelian algebra; and it is to these case that the word "additional" can be applied. We will see in fact that this is not generally the case for supersymmetric hierarchies.

Along with Definition 1.6, it is in practice convenient to have a "working definition" that is more suitable for computation. Our working definition is motivated by the following fact. The flows $\partial_{W^{\prime}(\Gamma)}$ generated via (1.5) by differential operators $\Gamma$ satisfying

$$
\left[\partial_{i}-\partial^{2}, \Gamma\right]=0
$$

commute with the KP flows. Indeed following [8] we have that

$$
\begin{aligned}
{\left[\partial_{W^{\prime}(\Gamma)}, \partial_{i}\right] } & =-\left[\partial_{W^{\prime}(\Gamma)}, \partial_{W^{\prime}\left(\partial^{\imath}\right)}\right] \\
& =-\partial_{W^{\prime}\left(\left[\Gamma, \partial^{i}\right]_{\phi}\right)},
\end{aligned}
$$


where $\left[\Gamma, \partial^{2}\right]_{\phi}$ is a modified Lie bracket (see Sect. 3) given by

$$
\begin{aligned}
{\left[\Gamma, \partial^{\imath}\right]_{\phi} } & =\partial_{W^{\prime}(\Gamma)} \partial^{\imath}-\partial_{W^{\prime}\left(\partial^{\imath}\right)} \Gamma+\left[\Gamma, \partial^{i}\right] \\
& =\left[\partial_{\imath}, \Gamma\right]+\left[\Gamma, \partial^{\imath}\right] \\
& =\left[\partial_{i}-\partial^{i}, \Gamma\right] \\
& =0
\end{aligned}
$$

We therefore call "additional symmetries" the flows generated by operators $\Gamma$ subject to (1.7). It is conceivable that (1.7) is also a necessary condition - that is, that all additional symmetries arise in this fashion; but we shall not attempt to prove it here.

This means that looking for the additional symmetries comes down to trying to find solutions for (1.7). An obvious solution to this equation is simply $\Gamma=\partial$, which, introduced in (1.7) and after applying a dressing transformation, gives precisely the KP flows (1.2). This agrees with the fact that the KP flows commute with each other.

A more interesting solution can be obtained if we allow for an explicit dependence on the time parameters of the hierarchy; that is, if we extend our ring of functions by the infinite set of independent variables $\left\{t_{1}, t_{2}, \ldots\right\}$, in which case we have to extend the derivative operator $\partial$ as a derivation in this new ring.

A priori, since $x$ and all the $t_{i}$ are independent variables of our infinite set of partial differential equations, we can automatically conclude that $\partial$ has to be extended trivially to the new ring. Nevertheless in the case KP, since the first flow (for dressable $L$ ) reads $\partial_{1} L=\left[L_{+}, L\right]=[\partial, L]$, and therefore gives $\partial=\partial_{1}$, one can identify $x$ with $t_{1}$. One can then define (see, for instance, [11]) a formal infinite-order differential operator

$$
\Gamma=\sum_{j \geq 1} j t_{j} \partial^{j-1}
$$

which satisfies (1.7), and from it a two-parameter family of flows

$$
\partial_{m, k} \phi=\left(\phi \Gamma^{k} \partial^{m} \phi^{-1}\right)_{-} \phi,
$$

that satisfy $\left[\partial_{m, k}, \partial_{n}\right]=0$. Notice that for $k=0$ and $m>0$ they agree with the KP flows. Moreover since $[\partial, \Gamma]=1$ it follows that the Lie algebra generated by $\Gamma^{k} \partial^{m}$, $k \geq 0$ and $m \in \mathbb{Z}$ is isomorphic (as a Lie algebra) to $\mathbf{W}_{1+\infty}$ and hence the algebra of additional symmetries is nothing but $\mathbf{W}_{\infty}$ (see [7] and [8]).

One can alternatively write the two-parameter family of flows in a Lax form

$$
\partial_{m, k} L=-\left[\left(M^{k} L^{m}\right)_{-}, L\right],
$$

where $M=\phi \Gamma \phi^{-1}$ is the dressed version of $\Gamma$. In this form, the additional symmetries prove to be a useful tool in the solution of the (multi-)matrix models for $2 d$ quantum gravity. Indeed, the partition function of the $(n-1)$-matrix model can be identified with the $\tau$-function of the $\mathrm{n}-\mathrm{KdV}$ hierarchy. The $\tau$-function is subject to an infinite set of $W$-constraints which follow from the string equation $[P, Q]=1$, where $Q$ is the $n$-th power of the Lax operator for the KP hierarchy and $P=\frac{1}{n}\left(M L^{-n+1}\right)_{+}$ is directly related to the generator $M$ of the additional symmetries [12].

In this paper we analyze the additional symmetries of several supersymmetric extensions of the KP hierarchy along these same lines. In order to do that we shall start in the second section with a brief review of a few basic facts about the supersymmetric formalism of pseudodifferential operators in order to introduce the basic objects we 
shall work with and to fix the notation. (For a more detailed account on this topic we send the reader to $[1,13,3,2]$.)

As we have seen in the case of the KP hierarchy, a very useful tool in the study of the additional symmetries is the map (1.4). We shall therefore need to define its supersymmetric version. We do that in the third section where we also prove that, analogously to the nonsupersymmetric case, it is a Lie algebra homomorphism.

In the fourth section we will consider the additional symmetries of the SKP hierarchy introduced by Manin and Radul (MRSKP). One of the distinctive features of this supersymmetric hierarchy is the fact that its algebra of flows is nonabelian and therefore not all the flows of the hierarchy are symmetries as well. Imposing an analogous condition to (1.7) we shall find the algebra of the additional symmetries to be isomorphic with the algebra of superdifferential operators SDOP. The additional symmetries of this particular hierarchy have been studied also in [9] and we find agreement with their results.

The even order SKP hierarchy will be discussed in the fifth section. An important feature of this hierarchy is the fact that it has only even Lax flows which can be represented in terms of an infinite set of even times. This has as a consequence the fact that not only the generator $Q$ of supertranslations but also the odd derivation $D$ generate symmetries of the hierarchy. As a result, the algebra of additional symmetries will be again seen to be the algebra SDOP of superdifferential operators.

Finally, in the sixth section we shall consider the Jacobian SKP hierarchy of Mulase and Rabin (JSKP), and we shall find its additional symmetries. In contrast to MRSKP, the flows of JSKP do commute with each other and the additional flows will therefore contain the JSKP flows as well. Moreover, we shall find that the symmetries form a Lie algebra isomorphic to the Lie algebra of superdifferential operators, which is in turn isomorphic - as a Lie algebra - with $\mathbf{S} \mathbf{W}_{1+\infty}$; the additional flows corresponding to a subalgebra containing $\mathbf{S} \mathbf{W}_{\infty}$.

The results seem to suggest that the additional symmetries considered so far are to a large extent kinematical in nature, although their explicit realization does depend on the dynamics.

\section{Supersymmetric Formalism}

Let $k$ be an arbitrary field of characteristic zero. We define our function space to be a $\mathbb{Z}_{2}$-graded ring over $k, R=R_{0} \oplus R_{1}$, endowed with an odd derivation $D$. Then $D^{2}=\partial$ is an even derivation. For the present purposes it suffices to restrict ourselves to the case in which $R$ is given by

$$
R=K \oplus K \theta,
$$

where $K$ is a subring of $k[[x]]$, the ring of formal power series in an even variable $x$, and $\theta$ is an odd variable satisfying $x \theta=\theta x$ and $\theta^{2}=0$. This gives rise to a structure of supercommutative algebra in the ring $R$. The $\mathbb{Z}_{2}$-grading is defined by putting $|x|=0$ and $|\theta|=1$ so that any element of $R_{0}$ (respectively $R_{1}$ ) is homogeneous of degree 0 (respectively 1 ). The odd derivation operator is given by $D=\partial_{\theta}+\theta \partial$ and satisfies the supersymmetric analog of the Leibniz rule

$$
D(a b)=D(a) b+(-)^{|a|} a D(b)
$$

where $a$ is a homogeneous element of $R$ of $\mathbb{Z}_{2}$-degree $|a|$ and $|D|=1$. We further define the ring of supersymmetric pseudodifferential operators (SWDO) with 
coefficients in $R$

$$
\mathscr{B} \equiv R\left(\left(D^{-1}\right)\right)=\left\{P=\sum_{\imath \gg-\infty} a_{i} D^{-i} \mid a_{i} \in R\right\} .
$$

The ring of SWDO's can be given the structure of a superalgebra using the generalized Leibniz rule

$$
D^{k} a=\sum_{i=0}^{\infty}\left[\begin{array}{c}
k \\
k-i
\end{array}\right](-1)^{|a|(k-\imath)} a^{[i]} D^{k-\imath}
$$
where $a$ is a homogeneous element of $R$ and $\left[\begin{array}{c}k \\ k-i\end{array}\right]$ are the so-called superbinomial
coefficients given by

$$
\left[\begin{array}{c}
k \\
k-i
\end{array}\right]=\left\{\begin{array}{c}
0 \\
\left(\begin{array}{c}
{\left[\frac{k}{2}\right]} \\
{\left[\frac{k-i}{2}\right]}
\end{array}\right) \text { for } i<0 \text { or }(k, i) \equiv(0,1) \bmod 2
\end{array}\right.
$$

Since the $\mathbb{Z}_{2}$-grading gets induced here we have that $\mathscr{R}=\mathscr{B}_{0} \oplus \mathscr{R}_{1}$, where

$$
\mathscr{R}_{0}=R_{0}\left(\left(D^{-1}\right)\right)=\left\{\sum_{i \gg-\infty} a_{i} D^{-i}|| a_{2 i}|=0,| a_{2 \imath+1} \mid=1\right\}
$$

and

$$
\mathscr{B}_{1}=R_{1}\left(\left(D^{-1}\right)\right)=\left\{\sum_{i \gg-\infty} a_{\imath} D^{-\imath}|| a_{2 i}|=1,| a_{2 i+1} \mid=0\right\}
$$

and we have thus defined the notion of an even (respectively odd) SWDO.

Let us remark the following fact:

$$
R\left(\left(D^{-1}\right)\right)=R\left(\left(\partial^{-1}\right)\right) \oplus R\left(\left(\partial^{-1}\right)\right) \partial_{\theta} .
$$

Indeed, on the one hand we clearly have $R\left(\left(\partial^{-1}\right)\right) \oplus R\left(\left(\partial^{-1}\right)\right) \partial_{\theta} \subset R\left(\left(D^{-1}\right)\right)$ since $D^{2}=\partial$ and $\partial_{\theta}=D-\theta D^{2}$. On the other hand any $\mathbf{S} \Psi D O$ can be written in the following manner:

$$
\begin{aligned}
\sum_{\imath} a_{i} D^{i} & =\sum_{\imath} a_{2 \imath} D^{2 \imath}+\sum_{i} a_{2 i+1} D^{2 \imath+1} \\
& =\sum_{i} a_{2 i} \partial^{i}+\sum_{\imath} a_{2 \imath+1} \partial^{\imath}\left(\partial_{\theta}+\theta \partial\right) \\
& \left.=\sum_{i} a_{2 \imath}+a_{2 \imath+1} \theta\right) \partial^{i}+\sum_{\imath} a_{2 i-1} \partial^{\imath} \partial_{\theta},
\end{aligned}
$$

so that we have also $R\left(\left(D^{-1}\right)\right) \subset R\left(\left(\partial^{-1}\right)\right) \oplus R\left(\left(\partial^{-1}\right)\right) \partial_{\theta}$.

In general it is important to distinguish in the ring of SWDO's the subring of supersymmetric differential operators SDOP

$$
\mathscr{R}_{+} \equiv R[D]=\left\{\sum_{0 \leq i \ll \infty} a_{\imath} D^{i} \mid a_{i} \in R\right\},
$$

with respect to which we have the splitting

$$
\mathscr{R}=\mathscr{R}_{+} \oplus \mathscr{R}_{-},
$$


where

$$
\mathscr{R}_{-} \equiv D^{-1} R\left[\left[D^{-1}\right]\right]=\left\{\sum_{i=1}^{\infty} a_{i} D^{-\imath} \mid a_{i} \in R\right\}
$$

denotes the integral SWDO's. If $P \in \mathscr{R}$ is any $\mathbf{S} \Psi \mathbf{D O}$ we shall denote by $P_{ \pm}$its projection onto $\mathscr{R}_{ \pm}$along $\mathscr{R}_{\mp}$.

The ring $\mathscr{R}$ of SWDO's can be made into a filtered associative $k$-algebra if we define

$$
\mathscr{B}^{n}=\left\{P=\sum_{\imath} a_{\imath} D^{i} \in \mathscr{R} \mid \text { ord } P \leq n\right\}
$$

where we say that ord $P=n$ if $a_{i}=0$ for all $i>n$ and $a_{n} \neq 0$. We have then

$$
\mathscr{R}^{n} \subset \mathscr{R}^{n+1} \text { and } \mathscr{R}=\bigcup_{n \in \mathbb{Z}} \mathscr{R}^{n}
$$

and under the multiplication $\mathscr{R}^{p} \times \mathscr{R}^{q} \rightarrow \mathscr{R}^{p+q}$. Moreover defining a bracket

$$
\text { [ ]: } \mathscr{B}^{p} \times \mathscr{R}^{q} \rightarrow \mathscr{B}^{p+q}
$$

via the graded commutator $[A B]=A B-(-)^{|A||B|} B A$, $\mathscr{B}$ becomes a Lie superalgebra.

Let us now consider the supersymmetric trace map Str $=\int \circ$ sres, where the supersymmetric noncommutative residue is given by sres $\left(\sum_{i} a_{i} D^{i}\right)=a_{-1}$ and the notion of integration can be defined abstractly as the canonical projection $\int: R \rightarrow$ $R / D R$, which simply means dropping the derivatives. One can alternatively consider the integral defined by the Berezinian, where for any homogeneous differential polynomial of $U=u+\theta v, f(U)=a(u, v)+\theta b(u, v), \int_{B} f(U)=\int b(u, v)$. The only difference between the two definitions consists in the fact that the abstract integration defined directly as a canonical projection is an even operation whereas the Berezinian has a $\mathbb{Z}_{2}$-degree of one.

The Str functional allows us now to define a dual pairing in $\mathscr{B}$ by

$$
\langle A, B\rangle \equiv \operatorname{Str} A B
$$

under which $R[D]$ and $D^{-1} R\left[\left[D^{-1}\right]\right]$ are maximally isotropic spaces nondegenerately paired with each other.

One of the central objects in our formalism is the space of sueprsymmetric Lax operators of degree $n$, defined by

$$
\mathfrak{M}_{n} \equiv\left\{L=D^{n}+\sum_{i=1}^{\infty} U_{i} D^{n-i}\left|U_{i} \in R,\right| U_{i} \mid \equiv i \bmod 2\right\} .
$$

(We shall drop the subscript $n$ whenever no confusion can arise.) Given any $L \in \mathfrak{M} \subset \mathscr{R}$ one can define $R_{L}$ the differential subring generated by the $U_{i}$ 's which will obviously induce the corresponding subrings $R_{L}[D] \subset R[D]$ and $R_{L}\left[D^{-1}\right] \subset R\left[D^{-1}\right] . \mathfrak{M}$ is an infinite-dimensional affine space modeled on the vector space $\mathscr{B}^{n-1}$ of SWDO's of order $n-1$. Its tangent space $T_{L} \mathfrak{M}$ at the point $L$ is thus isomorphic to $\mathscr{R}^{n-1}$ itself, namely

$$
T_{L} \mathfrak{M} \equiv\left\{A=\sum_{\imath=1}^{\infty} A_{i} D^{n-\imath}\left|A_{\imath} \in R,\right| A_{\imath}|\equiv| A \mid+n+i \bmod 2\right\} .
$$


Then to every tangent vector $A \in T_{L} \mathfrak{M}$ one can associate a vector field $D_{A}$ whose action on any $f \in R_{L}$ is defined by

$$
\begin{aligned}
D_{A} f & \left.\equiv \frac{d}{d \varepsilon} f\left(U_{i}+\varepsilon A_{\imath}\right)\right|_{\varepsilon=0} \\
& =\sum_{\imath=1}^{\infty} \sum_{k=0}^{\infty}(-)^{(|A|+n) k} A_{\imath}^{[k]} \frac{\partial f}{\partial U_{\imath}^{[k]}}
\end{aligned}
$$

where we do not impose a priori that $\varepsilon$ be even, i.e., $|L|=|A|$ is not necessarily true.

Lemma 2.19. $D_{A} D=(-)^{(|A|+n)} D D_{A}$.

The proof is a straightforward computation and it is left as an exercise.

Notice that $D_{A}: R_{L} \rightarrow R_{L}$ induces a map - also denoted $D_{A}$ with some abuse of notation $-D_{A}: R_{L} / D R_{L} \rightarrow R_{L} / D R_{L}$ given by $D_{A} \int f=\int D_{A} f$. But in this case since $R_{L} / D R_{L}$ is no longer a superalgebra, $D_{A}$ is no longer a derivation. This nevertheless will not affect the formalism.

In order to define the cotangent space of $\mathfrak{M}$ at $L$ it suffices to notice that the tangent space $T_{L} \mathfrak{M}$ is nondegenerately paired [via the pairing defined in (2.15)] with the quotient space $\mathscr{R} / D^{-n} \mathscr{R}_{-}$, and therefore we have

$$
T_{L}^{*} \mathfrak{M} \cong \mathscr{B} / D^{-n} \mathscr{R} \ldots
$$

\section{The Supersymmetric Radul Map}

In this section we shall introduce a supersymmetric generalization of the Radul map and we shall see that it defines a Lie algebra homomorphism between the space of SWDO's and $T_{L} \mathfrak{M}$. In order to do this we have first of all to define a Lie (super)algebra structure on $T_{L} \mathfrak{M}$. Of course, since the elements of $T_{L} \mathfrak{M}$ are in

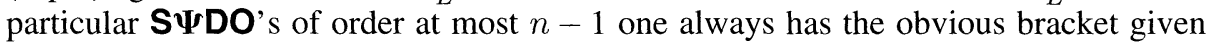
by the graded commutator. Still this is not the one that will allow us to exhibit the supersymmetric Radul map as a Lie algebra homomorphism. Instead let us consider the natural Lie bracket on vector fields on $\mathfrak{M}$, namely

$$
\left[D_{A}, D_{B}\right]=D_{A} D_{B}-(-)^{\left|D_{A}\right|\left|D_{B}\right|} D_{B} D_{A} \text {. }
$$

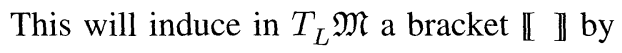

$$
\left[D_{A}, D_{B}\right]=D_{\llbracket A, B \rrbracket},
$$

whose explicit form we shall obtain now.

Lemma 3.3. The Lie bracket 【 I in $T_{L} \mathfrak{M}$ is given by

$$
\llbracket A, B \rrbracket=D_{A} B-(-)^{\left|D_{A}\right|\left|D_{B}\right|} D_{B} A,
$$

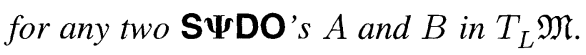

Proof. Consider $f$ an arbitrary function in $R_{L}$. Then

$$
\left[D_{A}, D_{B}\right] f=\left(D_{A} D_{B}-(-)^{\left|D_{A}\right|\left|D_{B}\right|} D_{B} D_{A}\right) f,
$$


which using the fact that $D_{A} f^{[k]}=(-)^{k\left|D_{A}\right|}\left(D_{A} f\right)^{[k]}$ (which follows by repeated application of Lemma 2.19) becomes

$$
\begin{aligned}
{\left[D_{A}, D_{B}\right] f=} & \sum_{i, j=1}^{\infty} \sum_{k, l=0}^{\infty}\left((-)^{k\left|D_{B}\right|+(l+k)\left|D_{A}\right|} A_{j}^{[l]} \frac{\partial B_{i}}{\partial U_{\jmath}^{[k]}}\right. \\
& \left.-(-)^{\left|D_{A}\right|\left|D_{B}\right|+k\left|D_{A}\right|+(l+k)\left|D_{B}\right|} B_{\jmath}^{[l]} \frac{\partial A_{i}}{\partial U_{j}^{[l]}}\right)^{[k]} \frac{\partial f}{\partial U_{i}^{[k]}} \\
= & \sum_{i=1}^{\infty} \sum_{k=0}^{\infty}(-)^{k\left(\left|D_{A}\right|+\left|D_{B}\right|\right)} \llbracket A, B \mathbb{\rrbracket}_{i}^{[k]} \frac{\partial f}{\partial U_{i}^{[k]}},
\end{aligned}
$$

where

$$
\llbracket A, B \rrbracket_{\imath}=\sum_{j=1}^{\infty} \sum_{l=0}^{\infty}(-)^{l\left|D_{A}\right|} A_{j}^{[l]} \frac{\partial B_{i}}{\partial U_{j}^{[k]}}-(-)^{\left|D_{A}\right|\left|D_{B}\right|+l\left|D_{B}\right|} B_{j}^{[l]} \frac{\partial A_{i}}{\partial U_{j}^{[l]}}
$$

and we get indeed (3.4).

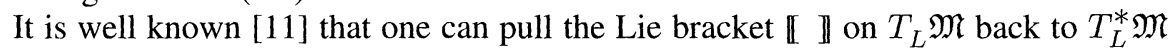
via the Adler map $J(X)=(L X)_{+} L-L(X L)_{+}$. In other words one can define a bracket []$_{L}^{*}$ on $T_{L}^{*} \mathfrak{M}$ such that

$$
\llbracket J(X), J(Y) \rrbracket=J\left([X, J]_{L}^{*}\right) .
$$

Computing $[X, Y]_{L}^{*}$ one finds [14]

$$
\begin{aligned}
{[X, Y]_{L}^{*}=} & (-)^{n(n+|X|)} D_{J(X)} Y+X(L Y)_{-} \\
& -\left((X L)_{+} Y\right)_{-}-(-)^{(n+|X|)(n+|Y|)}(X \leftrightarrow Y) .
\end{aligned}
$$

This already tells us that the Adler map is a Lie (super)algebra homomorphism mapping the cotangent space to the tangent space of $\mathfrak{M}$ at $L$, each of them being considered with the corresponding Lie algebra structure.

Now we can finally define the supersymmetric analog of the Radul map

$$
W: \text { SDOP } \rightarrow T_{L} \mathfrak{M}
$$

sending any $E \in$ SDOP to the tangent vector $W(E)$ defined by

$$
W(E) \equiv L E-\left(L E L^{-1}\right)_{+} L=\left(L E L^{-1}\right)_{-} L .
$$

Theorem 3.12. The supersymmetric Radul map is a Lie (super)algebra homomorphism, i.e.,

$$
\llbracket W(E), W(F) \rrbracket=W\left([E, F]_{L}\right)
$$

where $[E, F]_{L}$ is the modified Lie bracket on SDOP given by

$$
[E, F]_{L}=[E, F]+(-)^{n|E|} D_{W(E)} F-(-)^{|E||F|+n|F|} D_{W(F)} E
$$


Proof. By direct computation in the right-hand side we have

$$
\begin{aligned}
\llbracket W(E), W(F) \rrbracket \equiv & D_{W(E)} W(F)-(-)^{\left|D_{W(E)}\right|\left|D_{W(F)}\right|} D_{W(F)} W(E) \\
= & D_{W(E)}\left(L F L^{-1}\right)_{-} F-(-)^{\left|D_{W(E)}\right|\left|D_{W(F)}\right|}(E \leftrightarrow F) \\
= & \left(W(E) F L^{-1}\right)_{-} L+(-)^{n\left|D_{W(E)}\right|}\left(L D_{W(E)} F L^{-1}\right)_{-} L \\
& -(-)^{|F|\left|D_{W(E)}\right|}\left(L F L^{-1} W(E) L^{-1}\right)_{-} L \\
& +(-)^{|F|\left|D_{W(E)}\right|}\left(L F L^{-1}\right)_{-} W(E)-(-)^{\left|D_{W(E)}\right|\left|D_{W(F)}\right|}(E \leftrightarrow F) \\
= & \left(\left(L E L^{-1}\right)_{-} L F L^{-1}\right)_{-} L+(-)^{|E||F|}\left(\left(L F L^{-1}\right)_{-} L E L^{-1}\right)_{-} L \\
& -(-)^{|E||F|}\left(L F E L^{-1}\right)_{-} L+(-)^{n|E|}\left(L D_{W(E)} F L^{-1}\right)_{-} L \\
& -(-)^{\left|D_{W(E)}\right|\left|D_{W(\phi)}\right|}(E \leftrightarrow F) \\
= & \left(L[E, F] L^{-1}\right)_{-} L+(-)^{n|E|}\left(L D_{W(E)} F L^{-1}\right)_{-} L \\
& -(-)^{|F|(n+|E|)}\left(L D_{W(F)} E L^{-1}\right)_{-} L \\
= & W\left([E, F]_{L}\right) . \quad \square
\end{aligned}
$$

Remark 3.16. Notice that in the case where $E$ and $F$ are independent of $L$ we recover the usual Lie bracket on SDOP.

We have in this moment the following diagram where both maps $W$ and $J$ have been proven to be Lie algebra homomorphisms:

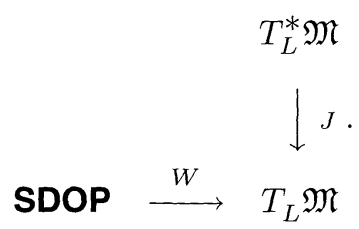

It would be thus interesting to see whether one can complete this diagram with a map $R$ such that $J \circ R=W$ and, if this is possible, to check whether the new map $R$ is a homomorphism as well.

We consider therefore the map $R:$ SDOP $\rightarrow T_{L}^{*} \mathfrak{M}$ defined by

$$
R(E)=-\left(E L^{-1}\right)_{-} \bmod D^{-n} \mathscr{R}{ }_{-},
$$

for any $E$ in SDOP. Since $D^{-n} \mathscr{B}_{-} \subseteq \operatorname{ker} J$ we have that indeed

$$
J \circ R(E)=W(E)
$$

for any $E$ in SDOP and therefore $J \circ R=W$.

Theorem 3.19. $R$ is a Lie algebra homomorphism, with

$$
[R(E), R(F)]_{L}^{*}=R\left([E, F]_{L}\right)
$$


Proof. Using the fact that $J \circ R=W$ and that $|R(E)|=|E|+n$ we have

$$
\begin{aligned}
{[R(E), R(F)]_{L}^{*}=} & -(-)^{n|E|} D_{W(E)}\left(F L^{-1}\right)_{-}+\left(E L^{-1}\right)_{-}\left(L\left(F L^{-1}\right)_{-}\right)_{-} \\
& -\left(\left(\left(E L^{-1}\right)_{-} L\right)_{+}\left(F L^{-1}\right)_{-}\right)_{-}-(-)^{|E||F|}(E \leftrightarrow F) \\
= & -(-)^{n|E|}\left(D_{W(E)} F L^{-1}\right)_{-}+(-)^{|E||F|}\left(F L^{-1} W(E) L^{-1}\right)_{-} \\
& +\left(E L^{-1}\right)_{-}\left(L F L^{-1}\right)_{-}-\left(E\left(F L^{-1}\right)_{-}\right)_{-} \\
& +\left(\left(E L^{-1}\right)_{+} L\left(F L^{-1}\right)_{-}\right)_{-}-(-)^{|E||F|}(E \leftrightarrow F) \\
= & -\left([E, F] L^{-1}\right)_{-}-(-)^{n|E|}\left(D_{W(E)} F L^{-1}\right)_{-} \\
& +(-)^{n|F|+|E||F|}\left(D_{W(F)} E L^{-1}\right)_{-} \\
= & R\left([E, F]_{L}\right) .
\end{aligned}
$$

Corollary 3.22. We have the following commutative diagram of Lie algebras:

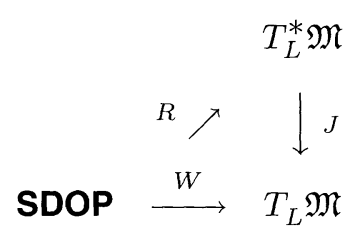

\section{Additional Symmetries of the MRSKP Hierarchy}

We shall start in this section the study of the additional symmetries of supersymmetric KP hierarchies by considering the supersymmetric extension of KP defined by Manin and Radul in [1], the MRSKP hierarchy.

One of the most remarkable features of the MRSKP hierarchy is the fact that it possesses a standard Lax formulation, although at the price that the (odd) flows do not commute with each other. It is due to its Lax formulation that this hierarchy has received the most attention from the physics community. Indeed, since the KP hierarchy is connected to $2 d$ quantum gravity via the Lax formalism, one might expect that MRSKP be relevant for $2 d$ quantum supergravity. So far, however, it seems that the relevant supersymmetric hierarchies in $2 d$ quantum supergravity are more or less naive supersymmetrizations of the KdV-type hierarchies [15, 16, 17].

In order to study its additional symmetries, let us first briefly recapitulate a few basic things about MRSKP that will be useful in the following. The MRSKP hierarchy is defined as the universal family of isospectral flows deforming a SWDO $\Lambda=D+\sum_{i \geq 1} U_{i} D^{1-i}$, with $U_{\imath} \in R$. But in contrast to the nonsupersymmetric case this infinite family of odd and even flows satisfy a nonabelian Lie superalgebra whose commutation relations are

$$
\left[D_{2 \imath}, D_{2 \jmath}\right]=0, \quad\left[D_{2 \imath}, D_{2 j-1}\right]=0, \quad\left[D_{2 \imath-1}, D_{2 j-1}\right]=-2 D_{2 i+2 j-2} .
$$

(We have adopted here the same sign conventions for the time parameters $t_{i}$ as in $[13,4]$.) A particular representation of (4.1) in terms of an infinite number of odd and 
even times $\left\{t_{1}, t_{2}, t_{3}, \ldots\right\}$ is given by

$$
\begin{aligned}
D_{2 i} & =\frac{\partial}{\partial t_{2 i}}, \\
D_{2 i-1} & =\frac{\partial}{\partial t_{2 \imath-1}}-\sum_{j>1} t_{2 j-1} \frac{\partial}{\partial t_{2 i+2 j-2}}
\end{aligned}
$$

where the odd times are odd variables satisfying $t_{2 i-1} t_{2 j-1}=-t_{2 \jmath-1} t_{2 \imath-1}$ and $t_{2 \imath-1}^{2}=0$. These flows are initially defined on $R$ but one can extend them on the whole $\mathscr{R}$ as evolutionary derivations, that is,

$$
\left[D_{2 \imath}, D\right]=\left[D_{2 i-1}, D\right]=0,
$$

and one can thus write the Lax flows of the MRSKP hierarchy in the following manner:

$$
\begin{aligned}
D_{2 i} \Lambda & =-\left[\Lambda_{-}^{2 \imath}, \Lambda\right]=\left[\Lambda_{+}^{2 i}, \Lambda\right] \\
D_{2 i-1} \Lambda & =-\left[\Lambda_{-}^{2 i-1}, \Lambda\right]=\left[\Lambda_{+}^{2 i-1}, \Lambda\right]-2 \Lambda^{2 \imath} .
\end{aligned}
$$

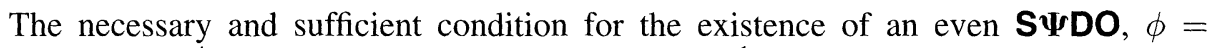
$1+\sum_{\geq 1} V_{i} D^{-i}$, with $V_{\imath} \in R$, such that $\Lambda=\phi D \phi^{-1}$ - that is, for $\Lambda$ to be dressable - is in this case $U_{1}^{[1]}+2 U_{2}=0$. If we restrict ourselves to such $\Lambda$ 's then we can alternatively define the MRSKP hierarchy as the family of flows on the dressing operator $\phi$

$$
D_{\imath} \phi=-\left(\phi D^{\imath} \phi^{-1}\right)_{-} \phi
$$

or equivalently

$$
\begin{aligned}
\frac{\partial \phi}{\partial t_{2 i}} & =-\left(\phi D^{2 i} \phi^{-1}\right)_{-} \phi \\
\frac{\partial \phi}{\partial t_{2 i-1}} & =-\left(\phi\left(D^{2 i-1}+\sum_{\jmath \geq 1} t_{2 j-1} D^{2 i+2 \jmath-2}\right) \phi^{-1}\right)_{-} \phi .
\end{aligned}
$$

Remark 4.7. One can prove that provided $\Lambda$ satisfies the dressability condition the two definitions of the MRSKP hierarchy are indeed equivalent. First of all it is obvious that given the flows on the dressing operator (4.5) one obtains the Lax flows on $\Lambda$ :

$$
\begin{aligned}
D_{i} \Lambda & =D_{\imath}\left(\phi D \phi^{-1}\right) \\
& =-\left(\phi D^{\imath} \phi^{-1}\right)_{-} \phi D \phi^{-1}+(-)^{2} \phi D \phi^{-1}\left(\phi D^{\imath} \phi^{-1}\right)_{-} \\
& =-\left[\Lambda_{-}^{i}, \Lambda\right] .
\end{aligned}
$$

In order to prove the converse let us introduce the dressed version of $\Lambda$ in the Lax flows and rewrite them in the following form:

$$
\left(D_{i} \phi+\left(\phi D^{\imath} \phi^{-1}\right)_{-} \phi\right) D \phi^{-1}-(-)^{\imath} \phi D \phi^{-1}\left(D_{i} \phi+\left(\phi D^{\imath} \phi^{-1}\right)_{-} \phi\right) \phi^{-1}=0 \text {. }
$$

Suppose now that $D_{i} \phi+\left(\phi D^{\imath} \phi^{-1}\right)_{-} \phi=A_{N} D^{N}+A_{N-1} D^{N-1}+\ldots$, for some arbitrary $N$. Then one obtains the following conditions for the leading coefficients:

$$
A_{N}=0 \text { for } N \text { odd },
$$


and

$$
\left.\begin{array}{c}
2 A_{N-1}-(-)^{n} A_{N}^{\prime}-(-)^{n} 2 V_{1} A_{N}=0 \\
A_{N-1}^{\prime}+2 V_{1} A_{N-1}-(-)^{n} V_{1}^{\prime} A_{N}=0
\end{array}\right\} \quad \text { for } N \text { even }
$$

That is, in both cases we obtain that - provided we drop the constants - the leading coefficient $A_{N}$ must vanish and hence (4.5) is satisfied.

Remark 4.11. Notice that one can dress the following obvious commutation relations:

$$
\begin{gathered}
{\left[D_{2 i}-D^{2 \imath}, D\right]=0} \\
{\left[D_{2 \imath-1}-D^{2 \imath-1}, D\right]=-2 D^{2 i}}
\end{gathered}
$$

and obtain the Lax flows (4.4).

The flows (4.5) are reminiscent of the supersymmetric Radul map (where in this case $n=1)$ and suggest us to define a map $W^{\prime}:$ SDOP $\rightarrow \mathscr{B}$ _ by

$$
W^{\prime}(E)=\left(\phi E \phi^{-1}\right)_{-} \phi
$$

for any SDOP $E$ such that $D_{n} \phi=-W^{\prime}\left(D^{n}\right)=-D_{W^{\prime}\left(D^{n}\right)} \phi$, where $D_{W^{\prime}\left(D^{n}\right)}$ is a flow on the Volterra group. The algebra of flows of MRSKP becomes in light of this definition a simple consequence of Theorem 3.12.

Proposition 4.15. The MRSKP flows satisfy the Lie superalgebra given in (4.1).

Proof. Following step by step the proof of Theorem 3.12 and replacing $L$ with $\phi$ we have that

$$
\left[D_{W^{\prime}(E)}, D_{W^{\prime}(F)}\right]=D_{W^{\prime}\left([E, F]_{\phi}\right)} .
$$

Applying this to our MRSKP flows we get for instance

$$
\begin{aligned}
{\left[D_{2 i-1}, D_{2 \jmath-1}\right] } & =\left[D_{W^{\prime}\left(D^{2 \imath-1}\right)}, D_{W^{\prime}\left(D^{2 \jmath-1}\right)}\right] \\
& =D_{W^{\prime}\left(2 D^{2 i+2 j-2}\right)} \\
& =-2 D_{2 \imath+2 \jmath-2} .
\end{aligned}
$$

One can in a similar way check that all the other commutators in (4.1) do indeed vanish.

After these general considerations concerning the MRSKP hierarchy we are now prepared to tackle the problem of finding its (additional) symmetries. We have seen that in the case of KP one defines a larger family of flows (i.e., containing the KP flows) which satisfy an algebra whose center is the KP hierarchy itself. Here the situation will turn out to be slightly different since the MRSKP flows themselves do not commute with each other but rather they obey the nonabelian (super)algebra (4.1).

Definition 4.18. We call (additional) symmetries of the MRSKP hierarchy the centralizer of the algebra (4.1) of flows of MRSKP in the algebra of times-dependent vector fields on $\mathfrak{M}$.

Analogous to the nonsupersymmetric case, one way to look for additional symmetries is to look for operators $\Gamma$ satisfying

$$
\left[D_{\imath}-D^{i}, \Gamma\right]=0 .
$$

The additional flow associated to $\Gamma$ is then obtained via the supersymmetric Radul map and is given by $D_{W^{\prime}(\Gamma)}$. 
One obvious solution is $\Gamma=\partial$, the even derivation on the ring $\mathscr{B}$ and the generator (via the Radul map) of the even flows of the hierarchy. Notice nevertheless that the odd derivation $D$ - the generator of the odd flows - does not obey (4.19) but for even $i$. One is therefore forced to conlcude that only the even flows are actually symmetries of the hierarchy, this being the most striking distinction from the nonsupersymmetric case. Apart from this "trivial" solution, one can of course ask whether there also exist time-dependent symmetries of the MRSKP hierarchy. The answer to this question is the object of the following lemma.

Lemma 4.20. Let $R\left[t_{\imath}\right]$ be the extension ring of $R$ by the time variables $\left\{t_{\imath}\right\}$ and let

$$
\begin{aligned}
& \Gamma_{0}=x+\frac{1}{2} \sum_{j \geq 1} j t_{j} D^{j-2}-\frac{1}{2} \sum_{j \geq 1} t_{2 j-1} \partial^{j-2} Q+\frac{1}{2} \sum_{i, j \geq 1}(i-j) t_{2 i-1} t_{2 j-1} \partial^{i+j-2} \\
& \Gamma_{1}=\theta+\sum_{j \geq 1} t_{2 j-1} \partial^{\jmath-1}
\end{aligned}
$$

where $Q=\partial_{\theta}-\theta \partial$, be formal infinite order (super)differential operators in $R\left[t_{i}\right] \llbracket D \rrbracket$ of $\mathbb{Z}_{2}$-degrees $\left|\Gamma_{0}\right|=0,\left|\Gamma_{1}\right|=1$. These operators enjoy the following properties:

(i) $\left[D_{\imath}-D^{i}, \Gamma_{0}\right]=0,\left[D_{\imath}-D^{i}, \Gamma_{1}\right]=0$, and $\left[D_{i}-D^{i}, Q\right]=0$ for any $i \geq 1$;

(ii) $\left[Q, \Gamma_{1}\right]=1,\left[Q, \Gamma_{0}\right]=-\Gamma_{1},\left[\partial, \Gamma_{0}\right]=1$;

(iii) $\left[\Gamma_{0}, \Gamma_{1}\right]=0,\left[\Gamma_{1}, \Gamma_{1}\right]=0,\left[\Gamma_{0}, \Gamma_{0}\right]=0$.

Proof. There is one point that ought to be mentioned here, concerning the extension of the derivations $\partial$ and $D$ to the ring $R\left[t_{2}\right]$. We recall that in the case of the KP hierarchy the first even time could be identified with $x$ because of the first flow which read $\partial_{1}=\partial$. Here, although the first even flow tells us again that $D_{2}=\partial$, things turn out to be different. Indeed $D$ cannot be (analogously to $\partial$ ) identified with $D_{1}$, as one can easily convince oneself by writing down the first odd flow. We are therefore forced to proceed safely and do not identify $x$ with $t_{2}$, but rather keep them as independent variables and extend trivially the action of $\partial$ and $D$ to the ring $R\left[t_{i}\right]$.

We can now define the "additional" flows of the MRSKP hierarchy as the following four-parameter family of odd and even flows:

$$
D_{m, k, l, p} \phi=W^{\prime}\left(\Gamma_{0}^{k} \Gamma_{1}^{l} Q^{p} \partial^{m}\right)
$$

with $k \geq 0, l=0,1, p=0,1$ and $m \in \mathbb{Z}$, where the even MRSKP flows can be obtained as a particular case, namely $D_{m, 0,0,0}=-D_{2 m}$ for $m>0$.

Theorem 4.22. The additional flows are symmetries of the MRSKP hierarchy, that is they commute with the MRSKP flows:

$$
\left[D_{i}, D_{m, k, l, p}\right]=0 .
$$

Proof. Using the expression of the flows in terms of the supersymmetric Radul map and Theorem 3.12 we have that

$$
\left[D_{\imath}, D_{m, k, l, p}\right]=D_{W^{\prime}\left(-\left[D^{\imath}, \Gamma_{0}^{k} \Gamma_{1}^{\prime} Q^{p} \partial^{m}\right]_{\phi}\right)},
$$

with

$$
\begin{aligned}
{\left[D^{i}, \Gamma_{0}^{k} \Gamma_{1}^{l} Q^{p} \partial^{m}\right]_{\phi}=} & D_{W^{\prime}\left(D^{i}\right)} \Gamma_{0}^{k} \Gamma_{1}^{l} Q^{p} \partial^{m}-(-)^{\imath(l+p)} D_{W^{\prime}\left(\Gamma_{0}^{k} \Gamma_{1}^{\prime} Q^{p} \partial^{m}\right)} D^{\imath} \\
& +\left[D^{\imath}, \Gamma_{0}^{k} \Gamma_{1}^{l} Q^{p} \partial^{m}\right] \\
= & -\left[D_{\imath}-D^{i}, \Gamma_{0}^{k} \Gamma_{1}^{l} Q^{p} \partial^{m}\right]
\end{aligned}
$$

which using Lemma 4.20 gives us the announced result. 
Corollary 4.26. The algebra of additional symmetries of the MRSKP hierarchy given by (4.21) is isomorphic to the Lie algebra of SDOP, which is isomorphic (as a Lie algebra) to $\mathbf{S w}_{1+\infty}$.

Proof. Indeed, the isomorphism is given by

$$
\begin{gathered}
z \mapsto-\partial, \quad \xi \mapsto Q+\Gamma_{1} \partial, \\
\partial_{z} \mapsto \Gamma_{0}, \quad \partial_{\xi} \mapsto \Gamma_{1} .
\end{gathered}
$$

The isomorphism between SDOP and $\mathbf{S W}_{1+\infty}$ is standard (see, e.g., [18]).

Remark 4.28. The fact that we have introduced the generator $Q$ of supertranslations may seem unsatisfactory to the purist, given that the MRSKP hierarchy is only defined in terms of abstract derivations $D_{i}$ and $D$. One could therefore ask whether it is really necessary to break manifest supersymmetric covariance in this fashion instead of trying to construct another even generator $\tilde{\Gamma}_{0}$ that would behave like $x$ and that would satisfy $\left[D_{i}-D^{i}, \tilde{\Gamma}_{0}\right]=0,\left[D, \tilde{\Gamma}_{0}\right]=\Gamma_{1}$, and $\left[\partial, \tilde{\Gamma}_{0}\right]=1$. This turns out to be impossible, essentially because $D$ itself is not a symmetry of the hierarchy. Indeed, an explicit calculation shows that

$$
\begin{aligned}
{\left[D_{2 i-1}-D^{2 \imath-1}, \Gamma_{1}\right] } & =\left[D_{2 i-1}-D^{2 \imath-1},\left[D, \tilde{\Gamma}_{0}\right]\right] \\
& =-2\left[\partial^{i}, \tilde{\Gamma}_{0}\right] \\
& =-2 i \partial^{i-1}
\end{aligned}
$$

which is different from zero and which thus contradicts the theorem. Hence such an operator $\tilde{\Gamma}_{0}$ cannot exist. One could nevertheless insist that the very definition of (additional) symmetry is not appropriate. Namely, one could argue that by the very nature of an integrable hierarchy, every flow of MRSKP should be thought of as a symmetry of all its other flows. In other words one should include $D$ too as a generator of the additional symmetries. This would of course require redefining the additional symmetries by adding to the previously found flows (4.21) the actual flows of the hierarchy. One could even go further and claim that once we allowed for the odd flows of the hierarchy to be part of the additional symmetries, what we have done is really to relax the condition (4.19) in order to include (4.13) as a particular case. But then consistency would force us to also look for possible times-dependent solutions of (4.19) where the right-hand side would be proportional with an appropriate power of $\partial$. If one carries on this computation one finds for instance a whole family of odd operators $\Gamma_{1}=\theta+\sum_{j \geq 1} a_{j} t_{2 j-1} \partial^{\jmath-1}$ satisfying

$$
\begin{aligned}
{\left[D_{2 i}-D^{2 \imath}, \Gamma_{1}\right] } & =0, \\
{\left[D_{2 \imath-1}-D^{2 i-1}, \Gamma_{1}\right] } & =\left(a_{i}-a_{1}\right) \partial^{i-1} .
\end{aligned}
$$

This embarrassment of riches suggests that this more relaxed notion of additional "symmetry" is of little interest.

\section{The $\operatorname{SKP}_{2}$ Hierarchy}

As it is well known the KP hierarchy can be thought of as a universal hierarchy for the series of generalized $\mathrm{KdV}$ hierarchies, in the sense that every $n$-th order $\mathrm{KdV}$ hierarchy is a reduction of KP, obtained by imposing $L_{-}^{n}=0$. Unfortunately this is no longer true for the MRSKP hierarchy. Imposing the constraint $L_{-}^{n}=0$ one can still 
get integrable hamiltonian hierarchies but not every generalized $n$-SKdV hierarchy is a reduction of MRSKP.

In a nutshell, this comes about because a superdifferential operator of order $n$ has a unique $n$-th root if and only if $n$ is odd. For even $n$, there may not exist an $n$-th root or, even if it exists, it need not be unique [1]. Nevertheless, the fact that for even $n$ a unique $\left(\frac{n}{2}\right)^{\text {nd }}$ root does always exist [2] has prompted the study of the so-called even order SKP hierarchy, $\mathrm{SKP}_{2}$, and it is the purpose of this section to study its additional symmetries.

$\mathrm{SKP}_{2}$ is defined [2] as the universal family of isospectral deformations of a S $\Psi D O$ of the form

$$
L=D^{2}+\sum_{i \geq 1} U_{\imath} D^{2-i}
$$

with $U_{i} \in R$ and its evolution is described by a commuting family of flows

$$
\partial_{\imath} L=-\left[L_{-}^{i}, L\right]=\left[L_{+}^{i}, L\right]
$$

where all the flows are even and therefore can be represented in terms of an infinite set of even times $\left\{t_{1}, t_{2}, \ldots\right\}$ by $\partial_{i}=\frac{\partial}{\partial t_{i}}$. In the following we shall restrict ourselves to operators $L$ which are dressable, i.e., which satisfy the conditions $U_{1}=U_{2}=0$.

Remark 5.3. Notice that one can dress the following obvious commutation relations

$$
\left[\partial_{n}-\partial^{n}, \partial\right]=0
$$

with an arbitrary $\phi=1+\sum_{\imath \geq 1} V_{i} D^{-\imath}, V_{\imath} \in R$, and obtain the $\mathrm{SKP}_{2}$ flows.

Let us now consider the problem of finding the additional symmetries for this hierarchy. Fortunately we can use our previous experience with KP and MRSKP to write down the generators of additional symmetries for $\mathrm{SKP}_{2}$. Indeed since the hierarchy has only even flows, it follows that the $x$-like generator for the additional flows of KP still commutes with the $\mathrm{SKP}_{2}$ flows. Moreover, and because of the same reason, both $D$ and $Q$ can now be considered generators of additional symmetries. In fact we have the following result:

Lemma 5.5. Let $R\left[t_{i}\right]$ be the extension ring of $R$ by the even time variables $\left\{t_{\imath}\right\}$ and let

$$
\Gamma=x+\sum_{t \geq 1} j t_{\jmath} \partial^{j-1}
$$

be a formal infinite order (super)differential operator in $R\left[t_{i}\right][[\partial]]$. This operator enjoys the following properties: $\left[\partial_{i}-\partial^{i}, \Gamma\right]=0$ and $[\partial, \Gamma]=1$. Moreover, the operators $D$ and $Q$ obey: $\left[\partial_{\imath}-\partial^{\imath}, D\right]=\left[\partial_{\imath}-\partial^{i}, Q\right]=0$.

We can now define the "additional" flows of the $\mathrm{SKP}_{2}$ hierarchy as the following four-parameter family of odd and even flows

$$
D_{m, k, l, p} \phi=W^{\prime}\left(\Gamma^{k} D^{l} Q^{p} \partial^{m}\right),
$$

with $k \geq 0, l=0,1, p=0,1$ and $m \in \mathbb{Z}$. Again, the original flows of the hierarchy can be obtained as a particular case, namely $D_{m .0,0,0}=-\partial_{m}$ for $m>0$. 
Theorem 5.7. The additional flows are symmetries of the $\mathrm{SKP}_{2}$ hierarchy, that is they commute with the MRSKP flows:

$$
\left[D_{\imath}, D_{m, k, l, p}\right]=0 .
$$

Proof. Using the expression of the flows in terms of the supersymmetric Radul map and Theorem 3.12 we have that

$$
\left[\partial_{\imath}, D_{m, k, l, p}\right]=D_{W^{\prime}\left(-\left[\partial^{2}, \Gamma^{k} D^{l} Q^{p} \partial^{m}\right]_{\phi}\right)},
$$

with

$$
\begin{aligned}
{\left[\partial^{\imath}, \Gamma^{k} D^{l} Q^{p} \partial^{m}\right]_{\phi} } & =D_{W^{\prime}\left(\partial^{\imath}\right)} \Gamma^{k} D^{l} Q^{p} \partial^{m}-D_{W^{\prime}\left(\Gamma^{k} D^{l} Q^{p} \partial^{m}\right)} \partial^{i}+\left[\partial^{\imath}, \Gamma^{k} D^{l} Q^{p} \partial_{m}\right] \\
& =-\left[\partial_{\imath}-\partial^{i}, \Gamma^{k} D^{l} Q^{p} \partial^{m}\right],
\end{aligned}
$$

which using Lemma 5.5 gives us the announced result.

Corollary 5.11. The algebra of additional symmetries of the $\mathrm{SKP}_{2}$ hierarchy given by (5.6) isomorphic to the Lie algebra of SDOP.

Proof. Indeed, the isomorphism is given by

$$
\begin{gathered}
z \mapsto-\partial, \quad \xi \mapsto \frac{1}{2}(D+Q), \\
\partial_{z} \mapsto \Gamma, \quad \partial_{\xi} \mapsto \frac{1}{2}(D-Q) \partial^{-1} .
\end{gathered}
$$

\section{Additional Symmetries of the JSKP Hierarchy}

Since there is no unique supersymmetric extension of the KP hierarchy one could of course ask what distinguishes the different supersymmetric KP hierarchies or which one of them is a more natural generalization of the KP hierarchy. We have previously argued that the MRSKP hierarchy has the advantage of possessing a Lax formulation. Nevertheless, from a geometrical point of view, it is not the MRSKP hierarchy, the one that seems the most natural supersymmetric generalization of the KP hierarchy. Indeed, according to [4], one can interpret geometrically the KP flows as deformations of a certain line bundle over a fixed manifold. This picture gets slightly modified in the supersymmetric case where the supersymmetry relation $D^{2}=\partial$ leads to deformations of the base supermanifold as well as of the line bundle on it. From this point of view the MRSKP hierarchy describes a special subset of deformations in which changes of the supermanifold are coupled to changes in the line bundle in such a way that the resulting hierarchy is integrable. It is the Jacobian SKP (JSKP) defined by Mulase and Rabin [3,4], the one that seems to provide the closest geometric analog of the KP hierarchy in the supersymmetric case since it only involves deformations in the line bundle.

With this motivation in mind let us define the JSKP hierarchy as the infinite family of odd and even commuting flows on the Volterra group given by

$$
\begin{aligned}
\frac{\partial \phi}{\partial t_{2 i}} & =-\left(\phi \partial^{i} \phi^{-1}\right)_{-} \phi, \\
\frac{\partial \phi}{\partial t_{2 i-1}} & =-\left(\phi \partial^{i-1} \partial_{\theta} \phi^{-1}\right)_{-} \phi
\end{aligned}
$$


where $\phi=1+\sum_{\geq 1} V_{i} D^{-i}$ and $\left\{t_{1}, t_{2}, t_{3}, \ldots\right\}$ is the same infinite set of odd and even times as in the case of the MRSKP hierarchy.

Remark 6.2. The even flows of JSKP coincide with the even flows of MRSKP being actually nothing but the original KP system. On the other hand, since $\phi \partial_{\theta} \neq \partial_{\theta} \phi$, it seems there is no simple way of writing the odd flows in terms of a Lax operator $L=\phi D \phi^{-1}$. We can nevertheless write the JSKP flows in a Lax form by defining $L=\phi \partial \phi^{-1}$ and $M=\phi \partial_{\theta} \phi^{-1}$, in terms of which the flows can be written as follows:

$$
\frac{\partial L}{\partial t_{2 \imath}}=-\left[L_{-}^{\imath}, L\right], \quad \frac{\partial L}{\partial t_{2 i-1}}=-\left[\left(L^{i-1} M\right)_{-}, L\right] .
$$

Following the same path as for MRSKP it is easily seen that the JSKP flows can be written in terms of the $W^{\prime}$ map, (4.14), by

$$
\begin{aligned}
D_{2 n} \phi & =-W^{\prime}\left(\partial^{n}\right)=-D_{W^{\prime}\left(\partial^{n}\right)} \phi, \\
D_{2 n-1} \phi & =-W^{\prime}\left(\partial^{n-1} \partial_{\theta}\right)=-D_{W^{\prime}\left(\partial^{n-1} \partial_{\theta}\right)} \phi .
\end{aligned}
$$

Proposition 6.5. The JSKP flows satisfy a commutative Lie superalgebra.

Proof. This is already clear since $\left[\partial^{n}, \partial^{m}\right]=\left[\partial^{n}, \partial^{m} \partial_{\theta}\right]=\left[\partial^{n} \partial_{\theta}, \partial^{m} \partial_{\theta}\right]=0$.

Lemma 6.6. Let $R\left[t_{i}\right]$ be the extension ring of $R$ by the time variables $\left\{t_{i}\right\}$ and let

$$
\begin{aligned}
& \Gamma_{0}=x+\sum_{\jmath \geq 1} j t_{2 j} \partial^{\jmath-1}+\sum_{\jmath \geq 1} j t_{2 \jmath+1} \partial^{j-1} \partial_{\theta} \\
& \Gamma_{1}=\theta+\sum_{\jmath \geq 1} t_{2 \jmath-1} \partial^{j-1} \\
& \Gamma_{2}=x \partial_{\theta}+\sum_{j \geq 1} j t_{2 \jmath} \partial^{\jmath-1} \partial_{\theta}
\end{aligned}
$$

be formal infinite order differential operators in $R\left[t_{2}\right]\left[\left[\partial, \partial_{\theta}\right]\right]$ of $\mathbb{Z}_{2}$-degrees $\left|\Gamma_{0}\right|=0$ and $\left|\Gamma_{1}\right|=\left|\Gamma_{2}\right|=1$. These operators have the following properties:

(i) $\left[D_{2 \imath}-\partial^{i}, \Gamma_{k}\right]=0$ and $\left[D_{2 i-1}-\partial^{2-1} \partial_{\theta}, \Gamma_{k}\right]=0$ for all $k=0,1,2$;

(ii) $\left[\partial, \Gamma_{1}\right]=0,\left[\partial_{\theta}, \Gamma_{1}\right]=1,\left[\Gamma_{1}, \Gamma_{1}\right]=0$;

(iii) $\left[\partial, \Gamma_{2}\right]=\partial_{\theta},\left[\partial_{\theta}, \Gamma_{2}\right]=0,\left[\Gamma_{2}, \Gamma_{2}\right]=0$;

(iv) $\left[\partial, \Gamma_{0}\right]=1,\left[\partial_{\theta}, \Gamma_{0}\right]=0,\left[\Gamma_{1}, \Gamma_{2}\right]=\Gamma_{0}$.

Proof. In order to compute the above commutators one has to extend the action of the derivatives $\partial$ and $\partial_{\theta}$ to the ring $R\left[t_{\imath}\right]$. Here too $\partial$ can be identified with the first even flow $D_{2}$ since

$$
D_{2} \phi=[\partial, \phi] .
$$

If we now consider the first odd flow

$$
D_{1} \phi=-\left(\phi \partial_{\theta} \phi^{-1}\right)_{-} \phi
$$

one may expect that $D_{1}$ can be identified with $\partial_{\theta}$. Still a short computation of the RHS of (6.11) shows us that this is not actually the case, but rather $D_{1}=\left[\partial_{\theta}, \phi\right]+V_{1} \phi$. It is therefore safe to extend trivially the action of $\partial$ and $\partial_{\theta}$ to $R\left[t_{\imath}\right]$. 
We can now define a three-parameter family of flows

$$
\begin{aligned}
D_{2 m, k, l} \phi & =W^{\prime}\left(\Gamma_{0}^{k} \Gamma_{1}^{l} \partial^{m}\right), \\
D_{2 m-1, k, l} \phi & =W^{\prime}\left(\Gamma_{0}^{k} \Gamma_{1}^{l} \partial^{m-1} \partial_{\theta}\right),
\end{aligned}
$$

where $k \geq 0, l=0,1$, and $m \in \mathbb{Z}$. Here the original JSKP flows are a special case, $D_{m, 0,0}=-D_{m}$ for $m>0$, whereas the other ones represent the additional symmetries of the JSKP hierarchy.

Theorem 6.13. The additional flows are symmetries of the JSKP hierarchy, in other words they commute with the flows on the Volterra group.

Proof. We only have to use Theorem 3.12 and we obtain, for instance, for the even flows

$$
\left[D_{2 \imath}, D_{2 m, k, l}\right]=-D_{W^{\prime}\left(\left[\partial^{2}, \Gamma_{0}^{k} \Gamma_{1}^{l} \partial^{m}\right]_{\phi}\right)}
$$

where

$$
\begin{aligned}
{\left[\partial^{i}, \Gamma_{0}^{k} \Gamma_{1}^{l} \partial^{m}\right]_{\phi} } & =D_{W^{\prime}\left(\partial^{\imath}\right)} \Gamma_{0}^{k} \Gamma_{1}^{l} \partial^{m}+\left[\partial^{i}, \Gamma_{0}^{k} \Gamma_{1}^{l} \partial^{m}\right] \\
& =-\left[D_{2 i}-\partial^{i}, \Gamma_{0}^{k} \Gamma_{1}^{l} \partial^{m}\right] \\
& =0
\end{aligned}
$$

Analogous computations give us that

$$
\begin{aligned}
{\left[D_{2 i}, D_{2 m-1, k, l}\right] } & =-D_{W^{\prime}\left(\left[\partial^{2}, \Gamma_{0}^{k} \Gamma_{1}^{l} \partial^{m-1} \partial_{\theta}\right]_{\phi}\right)}=0, \\
{\left[D_{2 i-1}, D_{2 m, k, l}\right] } & =-D_{W^{\prime}\left(\left[\partial^{\imath-1} \partial_{\theta}, \Gamma_{0}^{k} \Gamma_{1}^{l} \partial^{m}\right]_{\phi}\right)}=0, \\
{\left[D_{2 \imath-1}, D_{2 m-1, k, l}\right] } & =-D_{W^{\prime}\left(\left[\partial^{\imath-1} \partial_{\theta}, \Gamma_{0}^{k} \Gamma_{1}^{l} \partial^{m-1} \partial_{\theta}\right]_{\phi}\right)}=0,
\end{aligned}
$$

which finally proves the above statement.

Corollary 6.19. The Lie superalgebra of symmetries of the Jacobian SKP hierarchy is isomorphic to SDOP.

Proof. Let $A$ be the Lie superalgebra of symmetries given by (6.12). It is generated via the Radul map by $\Gamma_{l}^{k} \Gamma_{1}^{l} \partial^{m}$ and $\Gamma_{0}^{k} \Gamma_{1}^{l} \partial^{m-1} \partial_{\theta}$ for $k \geq 0, l=0,1$ and $m \in \mathbb{Z}$. The isomorphism SDOP $\rightarrow$ A is given explicitly by

$$
\begin{aligned}
z & \mapsto-\partial, \quad \xi \mapsto \partial_{\theta}, \\
\partial_{z} & \mapsto \Gamma_{0}, \quad \partial_{\xi} \mapsto \Gamma_{1} .
\end{aligned}
$$

Remark 6.21. Of the flows $D_{m, k, l}$ defined by (6.12), all but the $D_{m, 0,0}$ with $m>0$ are additional symmetries. These additional symmetries are isomorphic to the direct sum of $\mathbf{S} \mathbf{W}_{\infty}$ with the abelian algebra generated by the flows $D_{m, 0,0}$ with $m \leq 0$. These flows are present only because the JSKP hierarchy is defined on the supervolterra group. If, as in the KP hierarchy, JSKP were defined on the space of Lax operators, these extra flows would not be present; for they act trivially on $L=\phi \partial \phi^{-1}$.

The isomorphism of the additional symmetries of all three SKP hierarchies deserves a final comment. The picture that begins to emerge is that the additional symmetries, although realized dynamically with explicit dependence on the times, are actually a kinematical property of the dynamical system; that is, symmetries of the phase space in which they are defined. 
Acknowledgements. I am grateful to J. M. Figueroa-O'Farrill and E. Ramos for suggesting the problem, for many helpful discussions, and for carefully reading an earlier version of this manuscript. In addition, I would like to thank W. Nahm and V. Rittenberg for their kind support and encouragement. Last, but not least, I am thankful to the Physics Department of Queen Mary and Westfield College for their hospitality during the final stages of this work.

\section{References}

1. Manin, Yu.I., Radul, A.O.: Commun. Math. Phys, 98, 65 (1985)

2. Figueroa-O'Farrill, J.M., Mas, J., Ramos, E.: Rep. Math. Phys. 3, 479 (1991)

3. Mulase, M.: J. Diff. Geometry 34, 651 (1991)

4. Rabin, J.: Commun. Math. Phys. 137, 533 (1991)

5. Orlov, A.Yu., Shulman, E.I.: Lett. Math. Phys. 12, 171 (1986)

6. Dickey, L.A.: Additional symmetries of KP, Grassmannian, and the string equation. hepth/9204092; Part II hep-th/9210155

7. Aoyama, S., Kodama, Y.: Phys. Lett. 278B, 56 (1992)

8. Figueroa-O'Farrill, J.M., Ramos, E.: hep-th/9211036, Lett. math. Phys. 27, 223 (1993)

9. Das, A., Sezgin, E., Sin, S.J.: hep-th/9111054, Phys. Lett. 227B, 435 (1992)

10. Radul, A.O.: Sov. Phys. JETP Lett. 50, 371 (1989), Funct. Anal. Appl. 25, 25 (1991); Vaysburd, I., Radul, A.: Phys. Lett. 274B, 317 (1992)

11. Dickey, L.A.: Soliton equations and Hamiltonian systems. Advanced Series in Mathematical Physics, Vol. 12. Singapore: World Scientific, 1991

12. Di Francesco, P., Ginsparg, P., Zinn-Justin, J.: 2D gravity and random matrices. hep-th/9306153, to appear in Physics Reports

13. Mulase, M.: Invent. Math. 92, 1 (1988)

14. Figueroa-O'Farrill, J.M., Ramos, E.: Commun. Math. Phys. 145, 43 (1992)

15. Becker, K., Becker, M.: hep-th/9301017, Mod. Phys. Lett. A 8, 1205 (1993)

16. Figueroa-O'Farrill, J.M., Stanciu, S.: hep-th/9302057, Phys. Lett. 316B, 282 (1993)

17. Figueroa-O'Farrill, J.M., Stanciu, S.: hep-th/9303168, Mod. Phys. Lett. A 8, 2125 (1993)

18. Bergshoeff, E., Pope, C.N., Romans, L.J., Sezgin, E., Shen, X.: Phys. Lett. 245B, 447 (1990)

Communicated by M. Jimbo 
